\title{
Clinical Hypertension
}

\section{Korea hypertension fact sheet 2020: analysis of nationwide population-based data}

Hyeon Chang Kim ${ }^{1,2^{*}}$ D , So Mi Jemma Cho ${ }^{3}$, Hokyou Lee ${ }^{1,2}$, Hyeok-Hee Lee ${ }^{1,2}$, Jongmin Baek , Ji Eun Heo ${ }^{3}$ and for the Korean Society of Hypertension (KSH) - Hypertension Epidemiology Research Working Group

\begin{abstract}
Background: The Korean Society of Hypertension has published the Korea Hypertension Fact Sheet 2020 to provide an overview of the magnitude and management status of hypertension and their recent trends.

Methods: The Fact Sheets were based on the analyses of Korean adults aged 20 years or older of the 2007-2018 Korea National Health and Nutrition Examination Survey (KNHANES) and the 2002-2018 National Health Insurance Big Data (NHI-BD).

Results: Currently, the population average of systolic/diastolic blood pressure was $118 / 76 \mathrm{mmHg}$ in Korean adults aged 20 years or older showing little change in the recent decade. However, the number of people with hypertension increased steadily, exceeding 12.0 million. Indeed, the number of people diagnosed with hypertension increased from 3.0 million in 2002 to 9.7 million in 2018. During the same period, the number of people using antihypertensive medication increased from 2.5 million to 9.0 million, and the number of people adherent to treatment increased from 0.6 million to 6.5 million. Hypertension awareness, treatment, and control rates increased rapidly until 2007, but showed plateaued thereafter. In 2018, the awareness, treatment, and control rates of hypertension among all adults were 67,63 , and $47 \%$, respectively. However, the awareness and treatment rates were only 17 and 14\% among adults aged 20 to 39 years old with hypertension. Among patients treated for hypertension, $61 \%$ of them were also using glucose-lowering or lipid-lowering drugs. Among antihypertensive prescriptions, $41 \%$ of the patients received monotherapy, $43 \%$ received dual therapy, and $16 \%$ received triple or more therapy. The most commonly prescribed antihypertensive medication was angiotensin receptor blockers, followed by calcium channel blockers and diuretics.
\end{abstract}

Conclusion: To achieve further improvement in management of hypertension, we need to encourage awareness and treatment in young adults. It is required to develop tailored prevention and management strategies that are appropriate for and inclusive of various demographics.

Keywords: Hypertension, Prevalence, Awareness, Treatment, Control, Antihypertensive medication, Korea

\footnotetext{
* Correspondence: hckim@yuhs.ac

'Department of Preventive Medicine, Yonsei University College of Medicine, Seoul 03722, South Korea

${ }^{2}$ Department of Internal Medicine, Yonsei University College of Medicine, Seoul 03722, South Korea

Full list of author information is available at the end of the article
}

(c) The Author(s). 2021, corrected publication 2021. Open Access This article is licensed under a Creative Commons Attribution 4.0 International License, which permits use, sharing, adaptation, distribution and reproduction in any medium or format, as long as you give appropriate credit to the original author(s) and the source, provide a link to the Creative Commons licence, and indicate if changes were made. The images or other third party material in this article are included in the article's Creative Commons licence, unless indicated otherwise in a credit line to the material. If material is not included in the article's Creative Commons licence and your intended use is not permitted by statutory regulation or exceeds the permitted use, you will need to obtain permission directly from the copyright holder. To view a copy of this licence, visit http://creativecommons.org/ licenses/by/4.0/. The Creative Commons Public Domain Dedication waiver (http://creativecommons.org/publicdomain/zero/1. 0/) applies to the data made available in this article, unless otherwise stated in a credit line to the data. 


\section{Introduction}

High blood pressure is the most important modifiable risk factor yet also the biggest contributor to cardiovascular and cerebrovascular diseases $[1,2]$. In the twentyfirst century, cardiovascular and cerebrovascular diseases mortality accounts for nearly a half of all deaths in the developed regions and for one quarter in the developing regions [3]. The cardiovascular disease mortality rate has been decreasing in Korea; nonetheless, heart disease, cerebrovascular disease, and high blood pressure remain the second, fourth, and tenth in the causes of death [4]. Moreover, due to the rapid aging of the population and improved prognosis from cardiovascular event, the absolute number of patients with cardiovascular disease continues to increase. According to the National Health Insurance Service (NHIS) statistics in Korea, the estimated medical cost of treating hypertension was 3830 billion Korean Won, accounting for $4 \%$ of all medical expenses or $16 \%$ of medical expenses for chronic diseases [5]. Hitherto, controlling blood pressure is crucial not only to reduce the burden of disease at a societal level but also to improve the quality of life at an individual level. Continuous monitoring of hypertension prevalence and management status should be the first step in reducing its burden. To achieve this, the Korean Society of Hypertension had published its first Hypertension Fact Sheet in 2018 [6] and have been periodically updating it thereafter.

\section{Materials and methods}

The Korea Hypertension Fact Sheet 2020 analyzed two nationally representative datasets. The first one is the Korea National Health and Nutrition Examination Survey (KNHANES) from 1998 to 2018. The KNHA NES is a national surveillance system in Korea that assesses the health and nutritional status of noninstitutionalized Korean population since 1998 [7]. The second one is the National Health Insurance (NHI) Big Data from 2002 to 2018. Organized by the National Health Insurance Service (NHIS), the NHI Big Data contains socio-demographics, hospital claims with International Classification of Diseases, 10th Revision (ICD-10) coding, and mortality data of the entire population of Republic of Korea [8]. Previously, the Korea Hypertension Fact Sheet 2018 analyzed adults aged 30 years from the KNHANES data and people of all age in the NHI Big Data. This year, the Korea Hypertension Fact Sheet 2020 included adults aged 20 years or older from both datasets.

The English version of the "Korea Hypertension Fact Sheet 2020 " is attached as a supplementary material of this manuscript, and the Korean version is also available at http://www.koreanhypertension.org/reference/guide.

\section{Analysis of the KNHANES from 1998 to 2018}

There have been 7 rounds of KNHANES between 1998 and 2018. The first three KNHANES were conducted every 3 years: KNHANES I (1998), KNHANES II (2001), and KNHANES III (2005). Since 2007, each survey has consisted of 3 years: KNHANES-IV (2007-2009), KNHA NES VII (2010-2012), and KNHANES VII (2016-2018). A total of 153,797 adults (average 21,971 per each survey) was analyzed. Hypertension was defined as systolic blood pressure (SBP) $\geq 140 \mathrm{mmHg}$, diastolic blood pressure (DBP) $\geq 90 \mathrm{mmHg}$, or self-reported use of antihypertensive medication for the purpose of blood pressure control. Awareness rate was defined as the proportion of people with physician diagnosis of hypertension among all people with hypertension. Treatment rate was defined as the proportion of people using antihypertensive drugs for 20 days or more per month among all people with hypertension. Control rate was defined as the proportion of people with $\mathrm{SBP}<140 \mathrm{mmHg}$ and DBP $<90 \mathrm{mmHg}$ among 1) all people with hypertension and 2) people treated for hypertension [7, 9]. To evaluate the magnitude and management status of hypertension without the effects of population aging, agestandardized rates were calculated based on the demographics of the Korean population in 2005 according to the Population and Housing Census, Statistics Korea.

\section{Analysis of the NHI big data from 2002 to 2018}

A repeated cross-sectional analysis of 114 million cases of hypertension (assuming 1 case per calendar year if observed more than once) was conducted. While the KNHANES data analysis defined hypertension based on measured blood pressure levels and use of antihypertensive medication, the NHI big data analysis defined hypertension based on diagnosis codes, because the claim database did not have records of blood pressure measurements. Healthcare utilization was defined as at least one health insurance claim for diagnosis of essential hypertension (ICD-10: I10) each year. Treatment of hypertension was defined as at least one health insurance claim for hypertension diagnosis with antihypertensive drug prescription each year. Adherence to treatment was defined as receiving prescriptions of antihypertensive drugs $\geq 290$ days $(80 \%)$ each year. Antihypertensive drugs were classified into diuretics (DU; thiazide-related diuretics, loop diuretics), beta-blockers (BB), calcium channel blockers (CCB), angiotensin converting enzyme inhibitors (ACEi), angiotensin receptor blockers (ARB), potassium-sparing diuretics (PSD), or others (alpha-blockers, vasodilators, etc.). If the regimen of antihypertensive drug had switched in a year, one with the longest duration was selected as the representative prescription of the patient for the given year. Complication screening rates were calculated for blood test 
( $\geq 1$ serum creatinine test) and $\geq 1$ routine urinalysis and/ or urine microalbumin test each year, separately. The estimated hypertension burden and management status were additionally calculated for young adults aged 2039 years.

\section{Results \\ Magnitude of hypertension: based on the KNHANES}

The average blood pressure of Korean adults has decreased between 1998 and 2008, but there has been no significant change in the last 10 years. Population mean SBP/DBP level was $118 / 76 \mathrm{mmHg}$ for Korean adults aged 20 years or older and $119 / 77 \mathrm{mmHg}$ for adults aged 30 years or older (Supplement, page 9). Over the last 20 years, the age-standardized mean blood pressure levels have decreased yet without significant change in recent years. The age-standardized prevalence of hypertension also modestly decreased from $26.0 \%$ (men $29.6 \%$, women $22.3 \%$ ) in 1998 to $23.5 \%$ (men $28.0 \%$, women 18.6\%) in 2018 (Supplement, page 10). However, with the rapid aging of the population, the absolute number of people with hypertension has steadily increased; as of 2018, the number has exceeded 12 million. The prevalence of hypertension increased with age in both sex (Supplement, page 11). The sex-specific prevalence was higher in men until the sixth decade and, thereafter, higher in women.

\section{Hypertension management status: based on the KNHA NES}

In general, the hypertension management (awareness, treatment, and control rates) has improved significantly over the past two decades. In 2018, among adults aged 20 or older with hypertension, the awareness rate was $67 \%$, the treatment rate was $63 \%$, and the control rate was $47 \%$ (Supplement, page 5). However, the degree of management varied greatly by age. All management indices have improved significantly in older adults but not in younger adults. Among patients under age 50 with hypertension, awareness, treatment, and control rates remained below 50\%. (Supplement, pages 13-15).

\section{Healthcare utilization for hypertension: based on the NHI- big data}

The number of people diagnosed with hypertension has increased 3.2 times from 3 million in 2002 to 9.7 million in 2018. People receiving antihypertensive prescription has also increased 3.6 times from 2.5 million in 2002 to 9.0 million in 2018. More importantly, the number of people adherent to antihypertensive medication has increased more than tenfold from 0.6 million in 2002 to 6.5 million in 2018 (Supplement, page 18). The use of combination therapy has rapidly increased, with $40.7 \%$ using one class, $43.2 \%$ using two classes, and $16.1 \%$ using three or more classes of antihypertensive drug in 2018 (Supplement, page 20). In 2018, the most commonly prescribed antihypertensive drug class was ARB (71.4\%), followed by CCB (60.4\%), DU (25.8\%), BB (16.0\%), ACEi (2.0\%), and PSD (1.8\%), respectively (Supplement, page 21). ARB (47.3\%) and CCB (39.9\%) were commonly used for monotherapy, while ACEi/ARB plus $C C B$ was the most frequently prescribed dual therapy regimen (Supplement, page 22).

\section{Hypertension in young adults: based on the KNHANES and NHI-big data}

When extrapolating the KNHANES 2018 data to the entire Korean population, it is estimated that 1.27 million young adults (20-39 years) have hypertension. This accounts for nearly $10 \%$ of all adults with hypertension. However, the analysis of NHI-Big Data suggested that, among the 2451 thousand adults with undiagnosed hypertension, 959 thousand (39\%) of them were between the age 20 to 39 years (Supplement, page 25). According the KNHANES data from 1998 to 2018, the awareness and treatment rates of hypertension have been steadily rising in older adults but improved little in younger adults. Indeed, the awareness and treatment rates among adults age $20-39$ years with hypertension were 17.4 and $13.7 \%$, respectively (Supplement, page 26-27).

\section{Discussion}

The Korea Hypertension Fact Sheet 2020 provides an overview on the magnitude and management status of hypertension in Korea. Although the population average blood pressure and the prevalence of hypertension have changed little over the last decade, the absolute number of people with hypertension have increased steadily-now exceeding 12 million due to population aging. Hypertension management status in Korea had greatly improved until 2007 but remained stagnant thereafter. The greatest novelty of the Korea Hypertension Fact Sheet lies on its generalizability; the KNHANES provides unbiased sampling of the Korean population, and the NHI-Big Data provides medical service uses of the entire nation. However, there are some limitations to be addressed. First, the KNHANES is based on non-institutionalized residents of Korea; thus, it might not include people with severe diseases. Second, the exact collection methods and survey details varied across the KNHA NES. Despite standardized protocols and rigorous quality control procedures, such variability may have affected the analysis on secular trends. Third, the NHI-Big Data may not be optimal for identifying disease occurrence and prevalence, because the data have been collected for medical service claims and reimbursement purposes. Fourth, the adherence to antihypertensive medication was evaluated on a prescription basis. Thus, it is possible 
that adherence was overestimated, because we cannot ascertain whether the drug was actually taken or not. Fifth, we were limited to anonymized datasets; therefore, the data linkage between the two datasets was unachievable.

\section{Conclusions}

Despite the significant improvement in hypertension management over the past few decades, there is still room for further improvement. In particular, the hypertension management status was concerning in younger age groups, regardless of sex. To achieve further improvements in management, it is essential to enable early seeking for disease status and to encourage treatment in young adults. To prevent complications and death from hypertension, emphasizing the importance of treatment adherence and blood pressure control remains a top priority. We also need to develop tailored prevention and management strategies that are appropriate for and inclusive of various demographics.

\section{Supplementary Information}

The online version contains supplementary material available at https://doi. org/10.1186/s40885-021-00166-2.

Additional file 1.

\section{Acknowledgments}

The Korean Society of Hypertension - Hypertension Epidemiology Research Working Group thanks the Korea Disease Control and Prevention Agency (KDCA), and the National Health Insurance Service (NHIS) for providing data used for analysis.

\section{Consortium}

The Korean Society Hypertension (KSH) - Hypertension Epidemiology Research Working Group. Hyeon Chang Kim, Song Vogue Ahn, Sun Ha Jee, Sungha Park, Hae-Young Lee, Min Ho Shin, Sang-Hyun Ihm, Seung Won Lee, Hokyou Lee, Jong Ku Park, II Suh, Tae-Yong Lee, So Mi Jemma Cho, HyeokHee Lee, Jongmin Baek, Ji Eun Heo.

\footnotetext{
Authors' contributions

HCK contributed the conception and design of the study, acquisition of the data, interpretation of the analysis, and drafted the manuscript. SMJC, HL, $\mathrm{HHL}, \mathrm{JB}$, and JEH contributed the analysis and interpretation of data, and critical revision of the manuscript. All authors read and approved the final manuscript.
}

\section{Funding}

None.

\section{Availability of data and materials}

Additional file 1 Korea hypertension fact sheet 2020 - English version. The Korean version is also available at http://www.koreanhypertension.org/ reference/guide.

\section{Declarations}

\section{Ethics approval and consent to participate}

The study protocol was approved by the Institutional Review Board of Yonsei University Health System, Seoul, Korea (approval 4-2020-0207). Informed consent was waived, because this is a retrospective study of deidentified, routinely collected data.
Consent for publication

Not applicable. No individual data in any form is disclosed.

\section{Competing interests}

The authors declare that they have no competing interests.

\section{Author details}

${ }^{1}$ Department of Preventive Medicine, Yonsei University College of Medicine, Seoul 03722, South Korea. ²Department of Internal Medicine, Yonsei

University College of Medicine, Seoul 03722, South Korea. ${ }^{3}$ Department of

Public Health, Yonsei University Graduate School, Seoul 03722, South Korea.

Received: 29 December 2020 Accepted: 4 March 2021

Published online: 15 March 2021

References

1. Lopez AD, et al. Global and regional burden of disease and risk factors, 2001: systematic analysis of population health data. Lancet. 2006;367: 1747-57.

2. Feigin $\mathrm{VL}$, et al. Global burden of stroke and risk factors in 188 countries, during 1990-2013: a systematic analysis for the global burden of disease study 2013. Lancet Neurol. 2016:15:913-24.

3. Levenson JW, et al. Reducing the global burden of cardiovascular disease: the role of risk factors. Prev Cardiol. 2002;5:188-99.

4. Korea S. Causes of death statistics in 2019. Daejon: Statistics Korea; 2012.

5. National Health Insurance Service. The 2019 statistical yearbook on the usage of medical services by region. Wonju: National Health Insurance Service; 2020.

6. Korean Society Hypertension (KSH); Hypertension Epidemiology Research Working Group, Kim HC, Cho MC. Korea hypertension fact sheet 2018. Clin Hypertens. 2018;24:13.

7. Kweon S, et al. Data resource profile: the Korea National Health and nutrition examination survey (KNHANES). Int J Epidemiol. 2014:43:69-77.

8. Seong SC, et al. Data resource profile: the national health information database of the National Health Insurance Service in South Korea. Int J Epidemiol. 2016;46:799-800.

9. Hajjar I, Kotchen TA. Trends in prevalence, awareness, treatment, and control of hypertension in the United States, 1988-2000. JAMA. 2003;290(2): 199-206.

\section{Publisher's Note}

Springer Nature remains neutral with regard to jurisdictional claims in published maps and institutional affiliations.

Ready to submit your research? Choose BMC and benefit from:

- fast, convenient online submission

- thorough peer review by experienced researchers in your field

- rapid publication on acceptance

- support for research data, including large and complex data types

- gold Open Access which fosters wider collaboration and increased citations

- maximum visibility for your research: over $100 \mathrm{M}$ website views per year

At BMC, research is always in progress.

Learn more biomedcentral.com/submission 